\title{
Evaluation of systemic and mucosal immune responses in mice administered with novel recombinant Salmonella vaccines for avian pathogenic Esherichia coli
}

\author{
In-Gyeong Oh, John Hwa Lee* \\ College of Veterinary Medicine and Bio-Safety Research Institute, Chonbuk National University, Jeonju 561-756, Korea \\ (Received: January 4, 2013; Revised: June 17, 2013; Accepted: June 21, 2013)
}

\begin{abstract}
Avian pathogenic Escherichia coli (APEC) is a causative agent for a number of extra intestinal diseases and account for significant losses to the poultry industry. Since protective immunity against APEC is largely directed to virulence antigens, we have individually expressed four different viulence antigens, papA, papG, IutA, and CS31A, using an attenuated Salmonella Typhimurium and a plasmid pBB244. Following oral immunization of mice with combination of two or four of these strains, serum IgG and mucosal IgA responses were elicited against each antigen represented in the mixture. The antigen-specific mucosal IgA responses were significantly higher in the group of mice immunized with the heat-labile Escherichia coli enterotoxin B subunit (LTB) strain than those in the group of mice immunized without the LTB strain. While, there was no significant difference between these two groups in antigen-specific serum IgG responses. The results showed that LTB could act as mucosal immune adjuvant. To assess the nature of immunity, the distribution of antigen-specific IgG isotypes was analyzed. All groups promoted Th1-type immunity as determined by the $\mathrm{IgG} 2 \mathrm{a} / \mathrm{IgG} 1$ ratio. Thus, our findings provided evidence that immunization with a combination of several vaccine strains is one of the strategies of developing effective vaccines against APEC.
\end{abstract}

Keywords : Avian pathogenic Escherichia coli, heat-labile Escherichia coli enterotoxin B subunit, immune response, pBB244, Salmonella vaccine delivery

\section{Introduction}

Avian pathogenic Escherichia coli (APEC) is the most common secondary bacterial infection of commercial poultry flocks and may also be a primary pathogen. Disease syndromes caused by the Escherichia (E.) coli include both disseminated systemic conditions like colisepticemia and focal infections such as cellulitis [4, 5, 19, 23]. Many putative virulence factors of APEC have been known $[15,22]$ and among the factors, the CS31A surface antigen, encoded by the $c l p$ operon; P-fimbriae, encoded by the pap gene cluster (papA and papG); the aerobactin receptor, encoded by the gene liutA are the most common virulence factors found on APEC isolates in Korea.

Although antibiotic therapy is available to treat this infection, the development of an effective prophylactic or therapeutic vaccine will be of enormous benefit. The use of oral routes for immunization against infective diseases is desirable because oral vaccines are easier to administer and mucosal surfaces, on which immunity can be acquired by the routes, are the portals of entry for many pathogenic micro- bial agents [17, 25]. However, oral vaccine need more and repeated antigen doses to achieve the protective immune response level, therefore, it is necessary to developing strategies to identify mucosa-active immunostimulating agents, adjuvants [10].

Attenuated Salmonella strains have been developed as potential live oral vaccines against salmonellosis and as carriers for the delivery of heterologous antigens to the mammalian immune systems. The Salmonella strains secreting the antigens induced a more pronounced immune response than did the strain expressing the antigen in the cytosol $[2,7]$. A foreign antigen expression vector, plasmid pBP244 which was incorporated with the key components of the type-II Sec-dependent secretion system, has been constructed to improve the secretion of antigen proteins in the Salmonella vaccine system [13].

In this study, we have constructed an attenuated Salmonella $(S$.) Typhimurium vaccine system with pBB244 that can express the virulence factors of APEC and heat-labile Escherichia coli enterotoxin B subunit (LTB). We examined the possibility that an immunization with combination of

*Corresponding author

Tel: +82-63-270-2553, Fax: +82-63-270-3780

E-mail: johnhlee@chonbuk.ac.kr 
Table 1. Bacterial strains and plasmids used in this study

\begin{tabular}{|c|c|c|}
\hline Strain / Plasmid & Characteristic & References \\
\hline \multicolumn{3}{|l|}{ E. coli } \\
\hline M15 & E.coli host strain, kanamycine resistance & Qiagen \\
\hline JM109 & E.coli host strain & promega \\
\hline JOL718 & Wild type IutA $^{+} \mathrm{CS} 31 \mathrm{~A}^{+}$E.coli isolate from chicken & this study \\
\hline JOL708 & Top10 (containing pGEM T-easy expressing LTB) & this study \\
\hline JOL711 & Top10 (containing pET28a expressing papA) & this study \\
\hline JOL714 & M15 (containing pQE9 expressing papG) & this study \\
\hline JOL742 & M15 (containing pQE10 expressing IutA) & this study \\
\hline JOL483 & JM109 (containing pQE9 expressing CS31A) & this study \\
\hline \multicolumn{3}{|l|}{ S. Typhmurium } \\
\hline$\times 8501$ & hisG $\Delta c r p-28 \Delta a s d A 16$ & Guig and Curtiss [9] \\
\hline JOL401 & Wild type $S$.Typhimurium isolate from chicken & this study \\
\hline JOL912 & S. Typhimurium JOL $401 \Delta l o n \triangle c p x R$ AasdA16 & this study \\
\hline JOL924 & $\chi 8501$ (containing pBP244 and expressing papA) & this study \\
\hline JOL928 & JOL912 (containing pBP244 and expressing papG) & this study \\
\hline JOL930 & JOL912 (containing pBP244 and expressing IutA) & this study \\
\hline JOL954 & $\chi 8501$ (containing pBP244 and expressing CS31A) & this study \\
\hline JOL758 & $\chi 8501$ (containing pYA3560 and expressing LTB) & this study \\
\hline JOL921 & 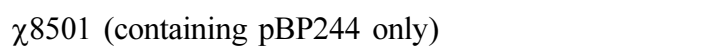 & this study \\
\hline \multicolumn{3}{|l|}{ Plasmids } \\
\hline pQE9 & Protein expression vector, ampicillin resistance & Qiagen \\
\hline pQE10 & Protein expression vector, ampicillin resistance & Qiagen \\
\hline pYA3560 & Asd $+;$ p15A ori & this study \\
\hline pBP244 & $\begin{array}{l}\text { Asd+; containing } \operatorname{Sec} A \text { (ATPase) } \\
\operatorname{Sec} B \text { (chaperone), LepB (signal peptidase) genes }\end{array}$ & Kim et al. [13] \\
\hline
\end{tabular}

E. coli: Escherichia coli, S. Typhmurium: Salmonella Typhmurium, LTB; heat-labile Escherichia coli enterotoxin B subunit.

these vaccines might elicit antigen-specific immune responses to every APEC antigen represented in the mixture. We also investigated the adjuvant effects of LTB with respect to mucosal and systemic immune responses of the vaccines.

\section{Materials and Methods}

\section{Bacterial strains, plasmids, media, and growth condi- tions}

The bacterial strains and plasmids used in this study are listed in Table 1. The $\Delta l o n \triangle c p x R$ AasdA16 attenuated $S$. Typhimurium, JOL912, were used as hosts. JOL912 were a derivative of JOL401, S. Typhimurium isolated from chicken. Plasmid pBP244 was used for extracelluar secretion of antigen protein. This vector was developed with the application of Sec-dependent protein secretion components, including LepB, SecA, and SecB proteins [13]. All strains were grown at $37^{\circ} \mathrm{C}$ in Luria-Bertani media (LB) (Difco, USA) with or without antibiotics. Diaminopimelic acid (DAP) (Sigma-Aldrich, USA) was added for the growth of $\mathrm{Asd}^{-}$strains [16]. Phosphate-buffered saline containing $0.01 \%$ gelatin (BSG) was used for the resuspension of the vaccine strains.
Construction of strains for protein expression and purification of proteins

Genes required for antigen proteins were obtained by polymerase chain reaction (PCR) using a pair of primers. Sequences of oligonucleotide primers used for PCR amplification and cloning are listed in Table 2. The amplified PCR products were cloned into the pET28a (Novagen, Germany), pQE9 or pQE10 (Qiagen, Germany). These expression plasmids were introduced into an E. coli Top10 (Invitrogen, USA) or M15 (Qiagen) or JM109 (Promega, USA). In-frame cloning was confirmed by nucleotide sequencing. The proteins were purified by an affinity purification process with $\mathrm{Ni}^{2+}$-nitrilotriacetic acid-agarose support (Qiagen). The purified proteins were verified by sodium dodecyl sulfate-polyacrylamide gel electrophoresis (SDS-PAGE), and protein concentrations were estimated by using Bradford kit (Bio Rad, USA).

Production of rabbit antisera against antigen proteins Hyperimmmune rabbit sera against papA, papG, IutA and CS31A were generated by subcutaneous immunization of NewZealand White rabbits with each purified protein. Each 
Table 2. Sequences of oligonucleotide primers used for polymerase chain reaction amplification and cloning

\begin{tabular}{ccl}
\hline \hline Gene & Primer & Sequence (5'-3') \\
\hline papA & $\mathrm{F}$ & CCGCGAATTCGCTCCAACTATTCCACAG \\
& $\mathrm{R}$ & CCCGCGTCGACTTACTGGTAACTTAAATT \\
$\operatorname{pap} G$ & $\mathrm{~F}$ & CCGCGAATTCATGAAAAAATGGTTCCCAGCTTTG \\
& $\mathrm{R}$ & CCCGCAAGCTTTTATGGCAATATCATGAGCAGCG \\
IutA & $\mathrm{F}$ & CGTGGATCCGCAACAAACCGATGATGAAACG \\
& $\mathrm{R}$ & CGGAAGCTTTCAGAACAGCACAGAGTAGTTCAGACC \\
\multirow{2}{*}{$\operatorname{ll} G$} & $\mathrm{~F}$ & CCGGAATTCATGTGGACCACTGGTGATTTAAT \\
& $\mathrm{R}$ & CCGAAGCTTTTAGTTATAAGTACTGCCA \\
\multirow{2}{*}{$e l t B$} & $\mathrm{~F}$ & CCGCGAATTCGCTCCCCAGTCTATTACAG \\
& $\mathrm{R}$ & CCGCAAGCTTCTAGTTTTCCATACTGATTG
\end{tabular}

Table 3. Experimental groups used in this study

\begin{tabular}{ll}
\hline \hline Group & Vaccine strain \\
\hline group A & JOL 924 (papA) + JOL 928 (papG) + JOL 758 (LTB) \\
group B & JOL 924+ JOL 928+ JOL 930 (IutA) + JOL954 (CS31A) + JOL 758 \\
group C & JOL 924 + JOL 928+ JOL 930+ JOL954 \\
group D & PBS \\
\hline
\end{tabular}

rabbit was primed with $250 \mu \mathrm{g}$ of purified protein antigen in Freud complete adujuvant (Sigma-Aldrich) and then followed booster injection with the same concentration of each protein in Freud incomplete adjuvant (Sigma-Aldrich) at 2 weeks later. The rabbits were bled through the marginal ear vein at 2 nd week after the last injection, then sera were obtained and stored at $-20^{\circ} \mathrm{C}$ until used.

\section{Construction of vaccine strains}

The genes for P-fimbriae (papA and papG), Aerobactin receptor (IutA), CS31A surface antigen $(\operatorname{clp} G)$ were cloned into the $\mathrm{Asd}^{+} \mathrm{pBP} 244$, respectively. The LTB were cloned into the $\mathrm{Asd}^{+} \mathrm{pYA3560}$. These cloned plasmids were introduced into the $\mathrm{Asd}^{-} \chi 6212$, to obtain the balanced lethal E.coli construct. The purified plasmids obtained from the $E$. coli constructs were then electroporated into an attenuated $S$. Typhimurium strain JOL912, and resulted in the strains, JOL924 (papA), JOL928 (papG), JOL930 (IutA), JOL954 (CS31A) and JOL758 (LTB), respectively. Selection for transformants was achieved by growth on LB agar plates without diaminopimelic acid supplementation. Only clones containing the recombinant plasmids were able to grow under these conditions. The expression of the antigen was assessed by immunoblot analysis.

\section{SDS-PAGE and immunoblot analyses}

SDS-PAGE and immunoblot analyses were performed by the same procedures as described in our previous study [18]. The secreted antigen proteins of vaccine strains were obtained from the cell-free culture supernatants of the TCAprecipitation process.

\section{Immunization and sample collection}

Overnight cultures of the vaccine strains were havested from LB broth and resuspended in BSG to total concentration of $3 \times 10^{9} \mathrm{CFU}$. Four groups of five inbred 7 weeks old female BALB/C mice were immunized orally with $20 \mu \mathrm{L}$ of the bacterial suspension. Immunization groups in this study were shown in Table 3. The group A of mice was immunized with a mixture of the vaccine strains including papA, pap $G$ and LTB strains. The mice in the group B were immunized with a mixture of the vaccine strains including papA, papG, IutA, CS31A and LTB strains, whereas the group C were immunized with a mixture of the vaccine strains including papA, papG, IutA and CS31A strains. Group D for the control received PBS only. Food and water were withdrawn $4 \mathrm{~h}$ prior to immunization and resupplied $30 \mathrm{~min}$ after immunization. Blood samples were obtained by retro-orbital puncture with heparinized capillary tubes at biweekly intervals. Sera were obtained from the whole blood by centrifugation at $4,000 \times \mathrm{g}$ for $5 \mathrm{~min}$ and stored at $-20^{\circ} \mathrm{C}$ until used. Vaginal secretions were collected by washing the vaginal tracts with $100 \mu \mathrm{L}$ of sterile PBS and stored at $-20^{\circ} \mathrm{C}$ until used [12]. The animal experiment described in this study was conducted with approval (CBU 2011-0017) from the Chonbuk National University Animal Ethics Committee in accordance with the guidelines of the Korean Council on Animal Care.

\section{Enzyme-linked immunosorbent assay (ELISA)}

Polystyrene 96-well flat-bottom microtiter plates were coated with $1 \mu \mathrm{g}$ of purified antigens per well and blocked with PBS containing 3\% skim milk at $4^{\circ} \mathrm{C}$ overnight. Vaginal secretions and sera were diluted $1: 4$ and $1: 100$, respec- 
tively. A $100 \mu \mathrm{L}$ volume of diluted sample was added to individual wells in duplicate and incubated for $1.5 \sim 2 \mathrm{~h}$ at $37^{\circ} \mathrm{C}$. Plates were treated with horseradish peroxidase-conju-

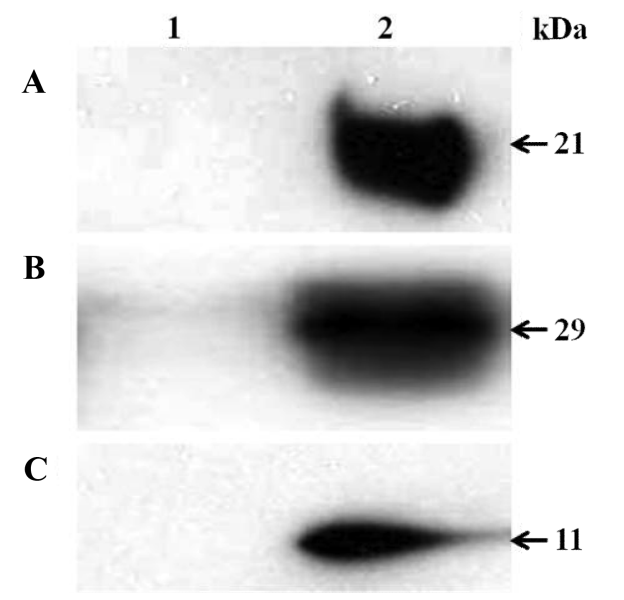

Fig. 1. (A)Immunoblot analysis of papA vaccine, (B) CS31A vaccine, $(\mathrm{C})$ LTB strain. Lane 1: vector control supernatant and lane 2: vaccine strain supernatant. papA (21 kDa), CS31A (29 $\mathrm{kDa})$, LTB $(11 \mathrm{kDa})$ proteins are indicated by arrows. gated goat anti-mouse IgG, IgG1, or IgG2a (Southern Biotechnology, USA) for sera and IgA for vaginal secretions. The reactions were visualized with $o$-phenylenediamine (SigmaAldrich) and stopped by $\mathrm{H}_{2} \mathrm{SO}_{4}$. The absorbance in each well was measured by an automated ELISA spectrophotometer (TECAN, Austria) at a wave length of $492 \mathrm{~nm}$. The concentrations of antibodies were determined by comparing the result with two concentrations of the standard immunoglobulin protein.

\section{Results}

Recombinant attenuated S. Typhimurium vaccines expressing papA, papG, IutA, CS31A and LTB.

Plasmid pBP244 was designed to effectively translocate antigens into extracellular space. The expression of proteins derived form Salmonella vaccines was confirmed by immunoblot analyses. The strains produced the papA, CS31A and LTB at approximate molecular weights of $21 \mathrm{kDa}, 29 \mathrm{kDa}$ and $11 \mathrm{kDa}$ respectively, and a large amount of these proteins were detected in the culture supernatant (Fig. 1). The pap $G$ and IutA proteins were barely detected in the supernatant and were mostly detected in the cell pellets.
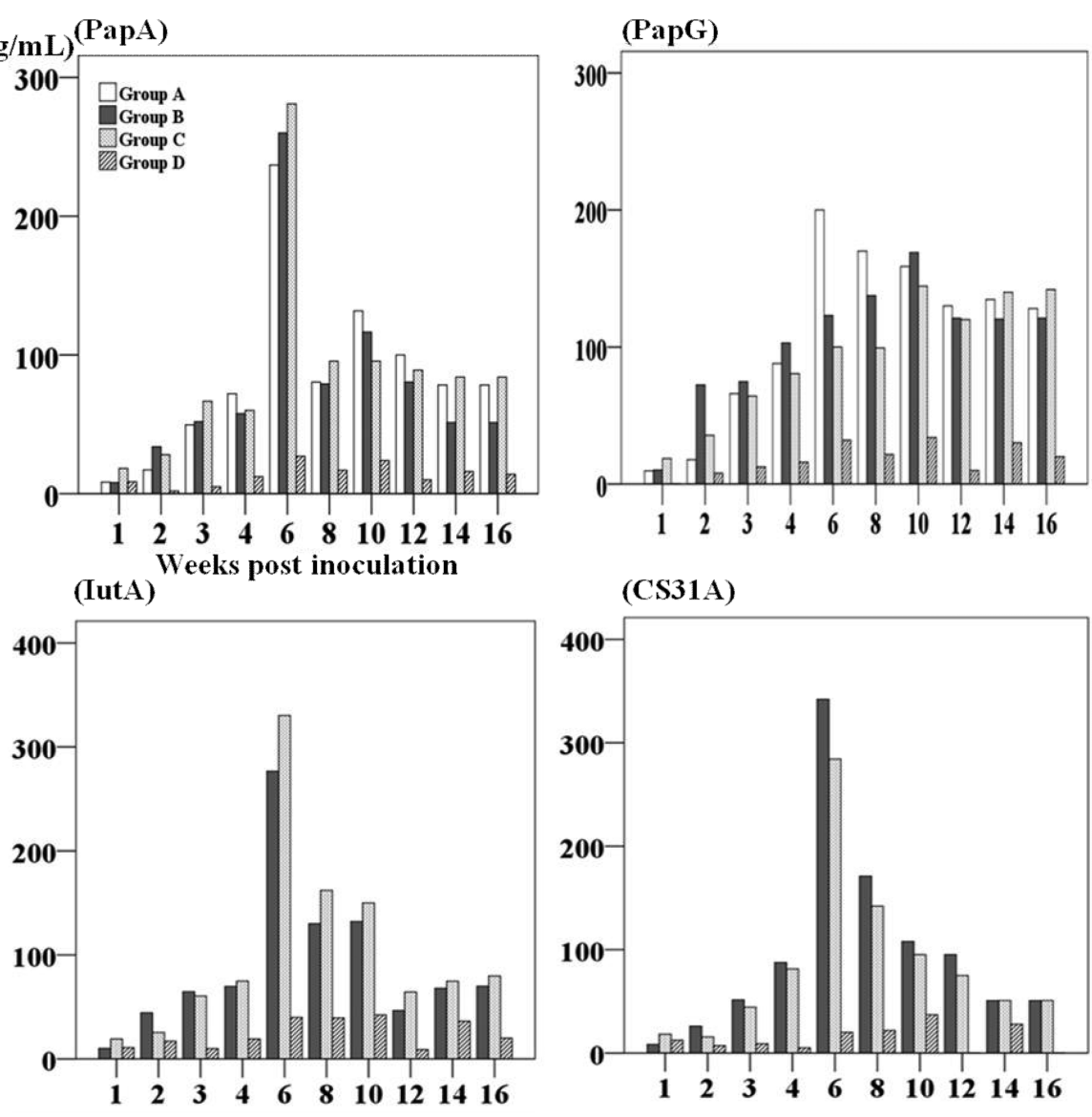

Fig. 2. Antigen-specific serum IgG responses. Antibody titers are shown as geometric mean titers for each group single oral immunization. Panel papA: serum IgG responses to papA, panel papG: serum IgG responses to papG, panel IutA: serum IgG responses to IutA, and panel CS31A: serum IgG responses to CS31A. 
(PapA)

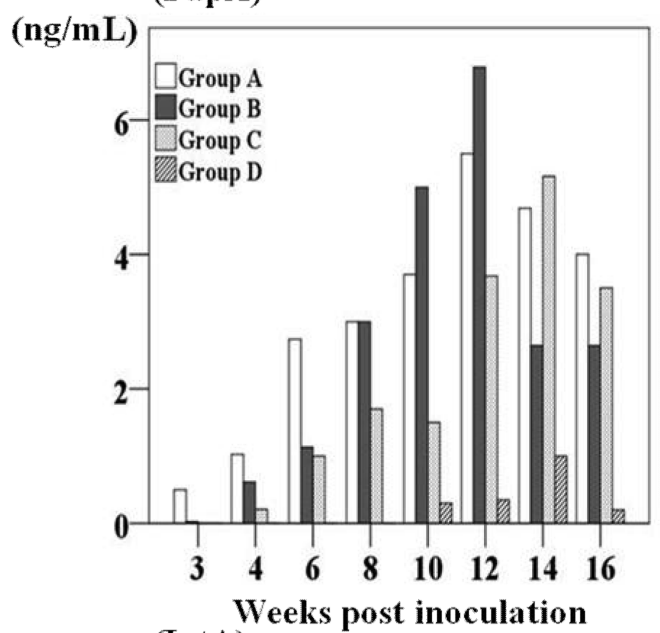

(IutA)

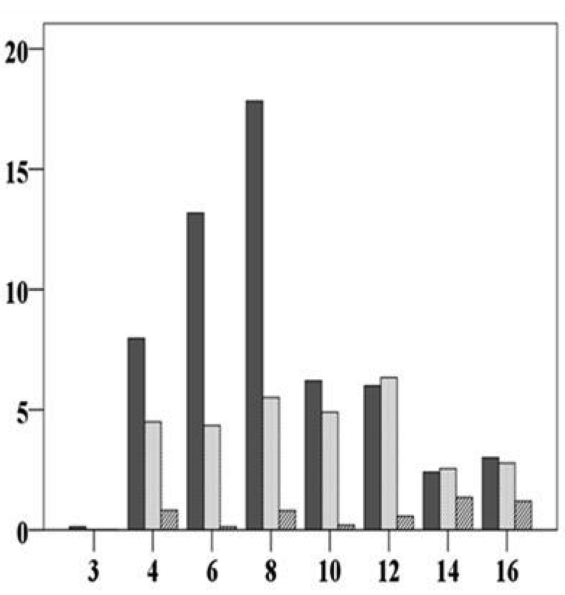

(PapG)

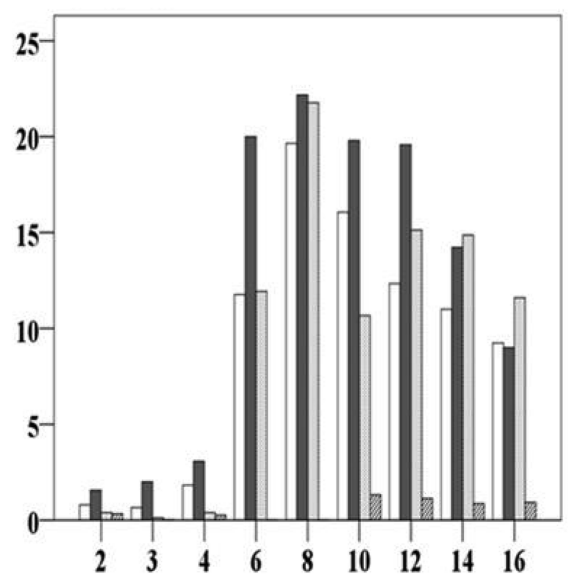

(CS31A)

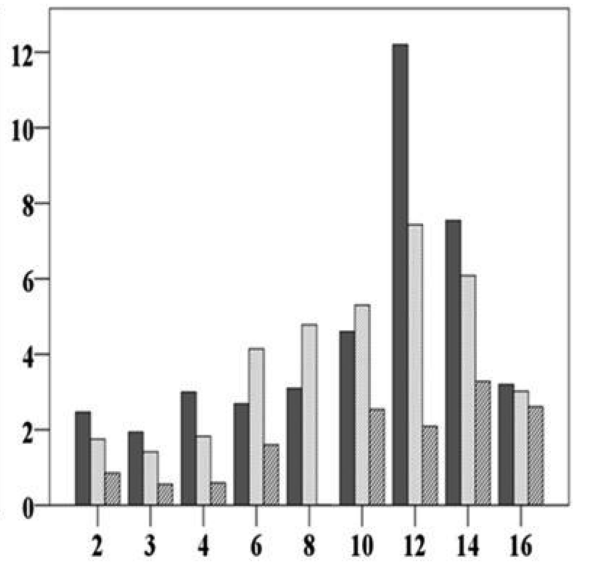

Fig. 3. Antigen-specific mucosal IgA responses. Antibody titers are shown as geometric mean titers for each group single oral immunization. Panel A: mucosal IgA responses to papA, panel B: mucosal IgA responses to papG, panel C: mucosal IgA responses to IutA, and panel D: mucosal IgA responses to CS31A.

\section{Systemic and mucosal immune responses induced by recombinant $S$. Typhimurium vaccines}

To evaluate the protective immune responses elicited by immunization with vaccine strains in combinations, BALB/c mice were immunized with a mixture of two or four vaccine strains. After immunization, the vaginal samples and sera were obtained at biweekly intervals to measure antigen-specific antibody responses. Adjuvant effects of recombinant LTB were also investigated. The antigen-specific serum IgG and mucosal $\operatorname{IgA}$ titers generated by immunization with vaccine strains were significantly higher than those by administration with PBS only. Serum anti-papA, -IutA, and CS31A IgG responses peaked at six weeks postimmunization, and the responses gradually declined. Serum papG-specific $\operatorname{IgG}$ responses increased slowly, and maximal levels detecded at 10 weeks postimmunization in group B and group C, immunization groups with four vaccine strains. Group A, immunization with two vaccine strains in the presence of the LTB, induced higher serum anti- papG IgG responses as com- pared to group B and group C. There was no significant difference between group B and group $\mathrm{C}$ in antigen-specific serum IgG responses (Fig. 2). In vaginal samples, however, antigen-specific IgA titers rose faster and higher in group B in the presence of LTB than in goup C in the absence of it. Maximal anti- $\operatorname{pap} G$ and -IutA levels were detected at 8 weeks postimmunization, while maximal anti- papA and CS31A levels were detected at 12 weeks postimmunization, and these gradually declined afterward (Fig. 3).

\section{IgG isotype analyses}

To further evaluate the nature of immunity following immunization, we determined the levels of $\mathrm{IgG}$ isotype subclasses IgG2a and IgG1 at 6 th week postimmunization. The IgG1 immune response was detected in lower than $100 \mathrm{ng} /$ $\mathrm{mL}$ titer, and data not shown. The IgG2a dominant immune responses were observed in all groups and the group B and group $\mathrm{C}$ produced more antigen-specific IgG2a isotype than the group A did (Fig. 4). 


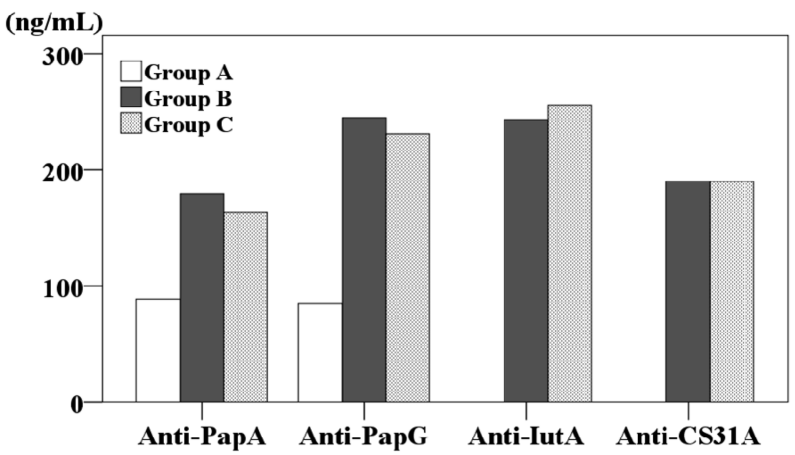

Fig. 4. Antigen-specific serum IgG2a responses. Serum IgG2a antibody titers are shown as geometric mean titers at 6 weeks.

\section{Discussion}

There are a variety of virulence-associated proteins of APEC playing important roles in the pathogenesis of colibacillosis. It has been proved that several virulence factors could be vaccine candidates $[8,14,20]$. However multiple virulence factors are produced by APEC strains and immune responses against one factor are not protective against APEC bearing different virulence factors. One of the strategies to overcome these is to combine different virulence factors in order to provide better protection. In this study, the recombinant attenuated Salmonella vaccines for papA, papG, IutA and CS31A virulence factors were constructed using the $S$. Typhimurium $\chi 8501$, JOL912 and the plasmid pBP244 [13] as a live vehicle for production and oral delivery of these virulence factors.

The antigen placed in the periplasm and extracellular fluid induced significantly higher levels of IgG than that mostly retained in the cytoplasm [11]. Immunoblot analyses showed that papA and CS31A proteins mostly detected in the culture supernatant, whereas papG and IutA protein barely detected in the supernatant and mostly detected in the cell pellet (data not shown). However, the ELISA results showed that the papG- and IutA-specific immune responses were of similar titer to the papA- and CS31A-specific immune responses regardless of the immunoblot data, which suggested that considerable amount of $p a p G$ and IutA proteins were located in the periplasm and induced the immune responses.

In this study, all immunized animals achieved high antibody titers against each of the guest APEC antigens, which demonstrated that immunization with a combination of vaccines elicits immune responses to every antigen respresented in the mix. The other studies [3] also reported that immunization with a combination of several virulence-associated proteins or their polyclonal antibodies might elicit better protection against pneumococcal infections.

Mucosal vaccination necessitates a suitable adjuvant, and LTB have been shown to enhance the immune response to co-administered antigens [6]. Our data showed that the antigen-specific mucosal IgA responses were significantly higher in the group of mice immunized with LTB than those in the group of mice immunized without LTB. Thus, this suggested that LTB could also be used as a mucosal immune adjuvant for these vaccines consisting of the APEC virulence factors.

In mice, T-helper type 1 (Th1) and Th2 cells stimulate antigen-specific B cells to secrete $\operatorname{IgG} 2 \mathrm{a}$ and $\mathrm{IgG} 1$, respectively [21]. The ratio of $\operatorname{IgG} 2 \mathrm{a}$ and $\operatorname{IgG} 1$ is therefore an indirect indication of the bias of the immune responses. In this study, immunization with the mixture of four strains expressing P-fimbriae, IutA and CS31A produced more $\operatorname{IgG} 2 \mathrm{a}$ isotype than immunization with the mixture of two strains expressing P-fimbriae only, which resulted in driving immunity biased to the Th1 pattern, which is related to cell mediated effector responses. Thus, the observation of Th1 type immune response may suggest that immunization with a combination of several virulence-associated vaccines is one of the strategies of developing effective vaccines for APEC, which survives inside macrophages and cell mediated immunity is more required to protect this pathogen [1, 24].

In conclusion, the construction of recombinant attenuated Salmonella vaccines expressing several virulence factors of APEC demonstrated that the immunization with the mixture of the vaccine strains is capable of eliciting both mucosal and systemic immune responses to every antigen represented in the mixture. LTB had effects on enhancing immunity in the mixture and can be used a mucosal immune adjuvant for the vaccines expressing APEC virulence factors.

\section{Acknowledgments}

This work was supported by Mid-career Researcher Program through National Research Foundation (NRF) grant funded by the Ministry of Education, Science and Technology (MEST) (No. 2012-R1A2A4A01002318), Korea.

\section{References}

1. Bastiani M, Vidotto MC, Horn F. An avian pathogenic Escherichia coli isolate induces caspase $3 / 7$ activation in J774 macrophages. FEMS Microbiol Lett 2005, 253, 133140.

2. Branger CG, Fetherston JD, Perry RD, Curtiss R 3rd. Oral vaccination with different antigens from Yersinia pestis KIM delivered by live attenuated Salmonella Typhimurium elicits a protective immune response against plague. Adv Exp Med Biol 2007, 603, 387-399.

3. Cao J, Chen D, Xu W, Chen T, Xu S, Luo J, Zhao Q, Liu B, Wang D, Zhang $X$, Shan $Y$, Yin Y. Enhanced protection against pneumococcal infection elicited by immunization with the combination of PspA, PspC, and ClpP. Vaccine 2007, 25, 4996-5005.

4. Dho-Moulin M, Fairbrother JM. Avian pathogenic Escherichia coli (APEC). Vet Res 1999, 30, 299-316.

5. Ewers C, Janssen T, Wieler LH. Avian pathogenic Escherichia coli (APEC). Berl Munch Tierarztl Wochenschr 2003, 116, 381-395.

6. Fingerut E, Gutter B, Goldway M, Eliahoo D, Pitcovski 
J. B subunit of E.coli enterotoxin as adjuvant and carrier in oral and skin vaccination. Vet Immunol Immunopathol 2006, 112, 253-263.

7. Galen JE, Levine MM. Can a 'flawless' live vector vaccine strain be engineered? Trends Microbiol 2001, 9, 372-376.

8. Gyimah JE, Panigrahy B, Wiliams JD. Immunogenicity of an Escherichia coli multivalent pilus vaccine in chickens. Avian Dis 1986, 30, 687-689.

9. Gulig PA, Curtiss R 3rd. Plasmid-associated virulence of Salmonella typhimurium. Infect Immun 1987, 55, 28912901.

10. Holmgren J, Czerkinsky C, Lycke N, Svennerholm AM. Mucosal immunity: implication for vaccine development. Immunobiology 1992, 184, 157-179.

11. Kang HY, Curtiss R 3rd. Immune responses dependent on antigen location in recombinant attenuated Salmonella typhimurium vaccines following oral immunization. FEMS Immunol Med Microbiol 2003, 37, 99-104.

12. Kang HY, Srinivasan J, Curtiss $\mathbf{R}$ 3rd. Immune responses to recombinant pneumococcal PspA antigen delivered by live attenuated Salmonella enterica serovar Typhimurium vaccine. Infect Immun 2002, 70, 1739-1749.

13. Kim SW, Kim YH, Yoo AY, Yu JE, Hur J, Lee J, Cha JH, Kang HY. Development of a protein secretion system with the application of sec-dependent protein secretion components. J Microbiol Biotechnol 2007, 17, 1316-1323.

14. Lynne AM, Foley SL, Nolan LK. Immune response to recombinant Escherichia coli Iss protein in poultry. Avian Dis 2006, 50, 273-276.

15. McPeake SJW, Smyth JA, Ball HJ. Characterization of avian pathogenic Escherichia coli (APEC) associated with colisepticaemia compared to faecal isolates from healthy birds. Vet Microbiol 2005, 110, 245-253.

16. Nakayama K, Kelly SM, Curtiss R 3rd. Construction of an $\mathrm{Asd}^{+}$expression-cloning vector: stable maintenance and high level expression of cloned genes in a Salmonella vaccine strain. Nat Biotechnol 1988, 6, 693-697.
17. Ogra PL, Faden H, Welliver RC. Vaccination strategies for mucosal immune responses. Clin Microbiol Rev 2001, 14, 430-445.

18. Oh IG, Moon BM, Lee JH, Hur J. Induction of systemic and mucosal immune responses in mice orally administered with recombinant attenuated Salmonella expressing subunits of P fimbriae of avian pathogenic Escherichia coli. J Vet Clin 2011, 28, 297-302.

19. Pourbakhsh SA, Boulianne M, Martineau-Doizé B, Fairbrother JM. Virulence mechanisms of avian fimbriated Escherichia coli in experimentally inoculated chickens. Vet Microbiol 1997, 58, 195-213.

20. Roland K, Karaca K, Sizemore D. Expression of Escherichia coli antigens in Salmonella typhimurium as a vaccine to prevent airsacculitis in chickens. Avian Dis 2004, 48, 595605.

21. Strindelius L, Filler M, Sjöholm I. Mucosal immunization with purified flagellin from Salmonella induces systemic and mucosal immune responses in $\mathrm{C} 3 \mathrm{H} / \mathrm{HeJ}$ mice. Vaccine 2004, 22, 3797-3808.

22. Stordeur P, Marlier D, Blanco J, Oswald E, Biet F, Dho-Moulin M, Mainil J. Examination of Escherichia coli from poultry for selected adhesin genes important in disease caused by mammalian pathogenic E.coli. Vet Microbiol 2002, 84, 231-241.

23. Vandemaele F, Assadzadeh A, Derijcke J, Vereecken M, Goddeeris BM. Avian pathogenic Escherichia coli (APEC). Tijdschr Diergeneeskd 2002, 127, 582-588.

24. Vindurampulle CJ, Cuberos LF, Barry EM, Pasetti MF, Levine MM. Recombinant Salmonella enterica serovar Typhi in a prime-boost strategy. Vaccine 2004, 22, 37443750.

25. Zhao X, Zhang M, Li Z, Frankel FR. Vaginal protection and immunity after oral immunization of mice with a novel vaccine strain of Listeria monocytogenes expressing human immunodeficiency virus type 1 gag. J Virol 2006, 80, 8880-8890. 